\title{
8×10 Gbps optical system with DCF and EDFA for different channel spacing
}

\author{
Saif H. Abdulwahed* and Tabarak M. Abass \\ Communication Engineering Department, Al- Hussein University College of Engineering, Iraq \\ Received: 02-March-2016; Revised: 23-May-2016; Accepted: 27-May-2016 \\ (C)2016 ACCENTS
}

\begin{abstract}
An optical system with specification of eight channels, 10 Gbps as bit rate, differential phase shift keying (DPSK) as a modulation format and $300 \mathrm{Km}$ optical fiber length that consist of five spans each with $60 \mathrm{Km}$ containing dispersion compensating fiber (DCF) and Erbium doped fiber amplifier (EDFA) have been studied. It is in the form of bit error rate (BER) Vs Signal-to-noise ratio (SNR) and quality $(Q)$-Factor Vs input power (P) for different channel spacing (200, 100, 50 and $25 \mathrm{GHz}$ ). The result pointed that the spacing between channels of $200 \mathrm{GHz}$ was the best because the BER has been improved by (2 orders) if it compared with the $100 \mathrm{GHz}$ channel spacing system at $10 \mathrm{~dB}$ SNR, the BER in the case of channel spacing $100 \mathrm{GHz}$ also improved by (1.9 orders) if it compared with the $50 \mathrm{GHz}$ channel spacing system at 10 dB SNR and the BER also improved by ( 0.7 orders) in the case of optical systems with $50 \mathrm{GHz}$ as if we compared it with system of $25 \mathrm{GHz}$ channel spacing at $10 \mathrm{~dB}$ SNR. The Q-Factor has been improved as the channel spacing increased, so generally the performance of an optical fiber system has been improved with the presence of DCF and EDFA in the optical link because the effect of these two elements in the linear and nonlinear fiber impairments.
\end{abstract}

\section{Keywords}

Dispersion compensating fiber (DCF), Erbium doped fiber amplifier (EDFA), Wavelength division multiplexed (WDM), Channel spacing.

\section{Introduction}

Dispersion of the transmitted optical signal causes distortion for both digital and analogue transmissions along optical fibers. When considering the major implementation of optical fiber transmission, which involves some form of digital modulation, then dispersion mechanisms within the fiber cause broadening of the transmitted light pulses as they travel along the channel [1].

A pulse can be broadened or compressed in a dispersive medium depending on the sign of the chirp and the dispersion. Dispersion compensation plays a key role in generating, amplification and propagation of femtosecond pulses. To obtain the ultra-short pulses, the pulse group delay (GD) should have about frequency independence after the dispersion compensation. Especially to compress a pulse to near the transform limit one should not only compensate the GD but also eliminate the high order dispersion term. [2]. Pulse distortion reduces maximum spacing between optical transmitters and receivers if the same BER performance of the system is to be maintained.

*Author for correspondence 100
When commercial single-mode optical fiber links were first introduced and installed, they were designed to offer zero dispersion at $13 \mu \mathrm{m}$, since that was the wavelength of commercially available light sources. Operated nowadays at $155 \mu \mathrm{m}$, these fibers exhibit substantial positive dispersion that may be cancelled out by using dispersion compensating fibers which provide large negative dispersion at that wavelength [3] [4].

Because of the relatively low transmission loss at wavelengths near $1550 \mathrm{NM}$ and the erbium-doped fiber amplifier (EDFA) working in this wavelength region, the preferred transmission window is around 1550 NM. A large number of the optical fiber cable transmission systems are installed based on standard single-mode fibers (SSMF), i.e., fibers with zero dispersion at $1310 \mathrm{NM}$ and a dispersion coefficient of about $17 \mathrm{ps} / \mathrm{NM} / \mathrm{km}$ at1550 NM [5]. Dispersion is one of the four basic limitations in the modern optical transmission systems. Pulse broadening occurs because of the nonzero bandwidth of an optical signal. Dispersion compensating optical fibers (DCF) with minus dispersion value and minus dispersion slope $(-50 \sim-500 \mathrm{ps} / \mathrm{nm} 2 / \mathrm{km})$ are used to compensate or optimize the dispersion characteristics of long distance transmission systems [6]. The high 
negative dispersion coefficient is provided by the DCF, which will be opposite in sign, but larger than the positive chromatic dispersion of conventional single mode fibers at $1550 \mathrm{NM}$. The technique based on DCF is widely developed in this connection to combat the chromatic dispersion of the fibers [7] [8].

Nonlinearity effects have to be considered when the optical communication systems operated at higher bit rates such as $10 \mathrm{Gbps}$ and above and/or at higher transmitter powers. In the case of WDM systems, nonlinear effects can become important, even at moderate powers and bitrates. The nonlinear effects that we consider in this section arise owing to the dependence of the refractive index of the intensity of the applied electric field, which in turn is proportional to the square of the field amplitude. At sufficiently high optical intensities, nonlinear refraction occurs in the core (Kerr effect), which is the variation of the index of refraction in light intensity. This makes nonlinear impairments a critical concern in optical networks for long-haul transmission commonly relies on high power lasers to transmit optical pulses over long spans to overcome attenuation. Nonlinear Impairments depend mainly on the fiber type and length and can be placed into two categories. The first includes the nonlinear effects that affect the energy of an optical pulse and includes:

- Stimulated Brillouin Scattering (SBS)

- Stimulated Raman Scattering (SRS)

- Four-wave mixing (FWM)

Nonlinear effects that affect the shape of an optical pulse include:

- Self-Phase Modulation (SPM)

- Cross-Phase Modulation (XPM) [9] [10]

As the optical fiber transmission systems evolved to longer distances and higher bit rates, the linear effect of fibers, which is the attenuation and dispersion, becomes the important limiting factor. As for WDM (Wavelength Division Multiplexed) systems that transmit multiple wavelengths simultaneously at even higher bit rates and distances, the nonlinear effects in the fiber begin to present a serious limitation. The success of high bit rate long haul point-to-point optical transmission networks depend upon how best the linear and nonlinear effects are managed. Several methods are reported for dispersion and nonlinearity management.

The EDFA (Erbium-doped fiber amplifiers) are the by far most important fiber amplifiers in the context of long-range optical fiber communications; they can efficiently amplify light in the $1.5-\mu \mathrm{m}$ wavelength region, where telecom fibers have their loss minimum. Since EDFA works in $1550 \mathrm{NM}$ wave band, the average single mode fiber (SMF) dispersion value in this wave band is very big, about 15-20ps / (NM. km-1) [11] [12].

\section{8×10 Gbps optical system design}

In this paper, we will discuss eight channel optical systems with different channel spacing, as shown in Figure 2, the optical transmitter has a DPSK (Differential phase shift keying) as a modulation format. An ITU-T G.652 optical fiber is used in the simulation to be the optimal fiber channel type against the fiber impairments as compared to other types of optical fibers. A dispersion compensating fiber (DCF) can be used to compensate the accumulated dispersion in the fiber which leads to decreasing the pulse broadening resulting from the chromatic dispersion (CD) effects. The DCF is one of the prime appliances to compensate chromatic dispersion for a large bandwidth of wavelengths. DCF is designed to reach large negative waveguide dispersion up to $-85 \mathrm{ps} / \mathrm{nm} . \mathrm{km}$, which enables to balance the amount of $\mathrm{CD}$ in the fiber.

As shown in Figure 1, the optical fiber channel consists of a DCF fiber with a length of $5 \mathrm{~km}$, its attenuation constant is $0.5 \mathrm{~dB} / \mathrm{km}$, the dispersion coefficient value is $-85 \mathrm{ps} / \mathrm{NM} / \mathrm{km}$ and the dispersion slope coefficient is $-0.3 \mathrm{ps} / \mathrm{nm} 2$. $\mathrm{km}$. The effective area of the DCF is $22 \mu \mathrm{m} 2$. The DCF is followed by an Erbium-Doped Fiber Amplifier EDFA with noise figure of $4 \mathrm{~dB}$ and Gain of $2.5 \mathrm{~dB}$ followed by a single mode fiber (SMF) with an attenuation value of $0.2 \mathrm{~dB} / \mathrm{km}$, a dispersion coefficient value of 17 $\mathrm{ps} / \mathrm{NM} / \mathrm{km}$ and dispersion slope coefficient of 0.075 $\mathrm{ps} / \mathrm{nm} 2 / \mathrm{km}$. The length of the SMF is $25 \mathrm{~km}$ with an effective area of $80 \mu \mathrm{m} 2$. The SMF is followed by an EDFA with Noise figure of $4 \mathrm{~dB}$ and a gain of $5 \mathrm{~dB}$. A second SMF, EDFA, DCF and EDFA with the same parameters of the first part are used again. This configuration forms the symmetrical dispersioncompensating scheme. The simulation value of the DGD parameter for all fiber types is $0.1 \mathrm{ps} / \mathrm{km}$. The span length is taken as $60 \mathrm{~km}$. Simulation parameters of the SMF and DCF are summarized in Table 1.

Then the signal is fed into the DPSK receiver to detect it and convert it into an electrical signal. A balanced receiver is used for the detection to convert the phase change into an intensity change. In order to analyze the received signal a set of eye diagrams was used. 


\section{Simulation results and discussion}

The system bit rate is 10 Gbps with DPSK as modulation format and with the use of DCF and EDFA we can observe that the system performance was improved in the case when the channel spacing was $200 \mathrm{GHz}$ if we compared it with the other cases gradually (100, 50 and $25 \mathrm{GHz}$ ) as shown through the bit error rate (BER) versus signal to noise ratio (SNR) curves and Q-factor versus input power (P) curves that are shown in Figures (3) and Figure (4). In the figure (Figure3), it is clear that the bit error rate (BER) is getting better with the increase of the SNR, so for example in the case of the channel spacing is $200 \mathrm{GHz}$ we can observe that if the signal to noise ratio (SNR) is $10 \mathrm{~dB}$, the BER is $10^{-8.2}$. While in the case of channel spacing is $100 \mathrm{GHz}$ the $\mathrm{BER}$ is $10^{-6.105}$. So the system performance was improved by $10^{2.095}$ orders. Again in SNR $10 \mathrm{~dB}$, The BER is $10^{-4.2}$ in case of channel spacing 50 $\mathrm{GHz}$, also the system has been improved by $10^{1.905}$ order if we compare between the 100 and $50 \mathrm{GHz}$ and so on with the other cases, shown in Table 2. It is well shown that the optical system with channel spacing of $200 \mathrm{GHz}$ is the best, because the dispersion is reduced, also Stimulated Raman Scattering (SRS), Cross-Phase Modulation (XPM) and Four-Wave Mixing (FWM) are also reduced. In Figure (4) there is the relation between the Q-factor and the input Power for each system. The highest channel spacing and the highest Q-factor which is the most improved.

Figures $(5,6)$ illustrate the spectrum of the optical signal before optical link and after it. So we can observe the nonlinear effects before and after the threshold point, so we take $0 \mathrm{dBm}$ and $9 \mathrm{dBm}$ as an example of two points one of them before the threshold point and the other after it.

\section{Conclusion}

DCF and EDFA are powerful elements that used in the fiber link and that's clear from their effects in reducing the optical impairments and improving the system performance and that is what we notice from Figure (3) and Figure (4). The BER of the system in case of channel spacing $200 \mathrm{GHz}$ is most improved compared to the other channel spacing. Also the quality factor (Q-factor) of the optical system was improved in the case of $200 \mathrm{GHz}$ channel spacing if we compared it with the other cases with increasing the input power to the threshold point reached the system performance begin to reduce as shown in Table 3. All this happens because the optical fiber link was almost dispersionless and almost clear from 102 fiber nonlinearities and that is the effect of the DCF and EDFA. Even the other cases of channel spacing $(100,50$ and $25 \mathrm{GHz})$ were considered better if we compared it with an optical system that has no DCF and EDFA.

Acknowledgment

None.

Conflicts of Interest

The authors have no conflicts of interest to declare.

\section{References}

[1] Senior JM, Jamro MY. Optical fiber communications: principles and practice. Pearson Education; 2009.

[2] Pal B. Frontiers in guided wave optics and optoelectronics. Intech Olajnica 19/2, 32000 Vukovar, Croatia, 2010.

[3] Poole CD, Wiesenfeld JM, Digiovanni DJ, Vengsarkar AM. Optical fiber-based dispersion compensation using higher order modes near cutoff. Journal of Lightwave Technology.1994;12(10):1746-58.

[4] Antos AJ, Smith DK. Design and characterization of dispersion compensating fiber based on the LP 01 mode. Journal of Lightwave Technology. 1994; 12(10):1739-45.

[5] Kristensen P, Andersen MN, Edvold B, Veng T, Grüner-Nielsen L. Dispersion and slope compensating module for G. 652 fiber with $\mathrm{x} 4$ reduced physical dimensions. In proceedings European conference on optical communication.We4P 2003 (p. 20).

[6] Gruner-Nielsen L, Edvold B, Magnussen D, Peckham D, Vengsarkar A, Jacobsen D, et al. Large volume manufacturing of dispersion compensating fibers. In optical fiber communication conference and exhibit, Technical Digest 1998 (pp. 24-5). IEEE.

[7] Grüner-Nielsen L, Knudsen SN, Edvold B, Magnussen D, Veng T, Larsen CC. Design and manufacture of dispersion compensating fibre for simultaneous compensation of dispersion and dispersion slope. In wavelength division multiplexing components 1999 (p. 134). Optical Society of America.

[8] Kaur M, Sarangal H, Bagga P. Dispersion compensation with dispersion compensating fibers (DCF). International Journal of Advanced Research in Computer and Communication Engineering.2015; 4(2):354-6.

[9] Darcie TE, Palais JC, Kaminow IP. Optical Communication. The Electrical Engineering Handbook. Ed. Richard C. Dorf Boca Raton: CRC Press LLC; 2000.

[10] Agrawal G. Nonlinear fiber optics and its applications in optical signal processing. Institute of Optics, University of Rochester, Rocheste, NY, United States.

[11] Kaler RS, Sharma AK, Kamal TS. Comparison of pre, post-and symmetrical-dispersion compensation schemes for $10 \mathrm{~Gb} / \mathrm{s}$ NRZ links using standard and 
dispersion compensated fibers. Optics Communications. 2002; 209(1):107-23.

[12] Tokle T, Davidson CR, Nissov M, Cai JX, Foursa D, Pilipetskii A. $6500 \mathrm{~km}$ transmission of RZ-DQPSK WDM signals. Electronics Letters. 2004; 40(7):1.

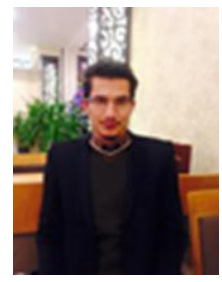

Saif Hussam Abdulwahed was born in Baghdad in 9/1/1990, he had a master's degree in communication engineering University of technology Baghdad and now he the repertoire of communication engineering department in Al-Hussein University college, member of the Society of Scientific \&

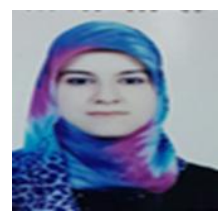

Tabarak Mohammed Abass was born in Ottawa, Canada in 15/4/1994, she is a student in communication engineering department in Al-Hussein University college, in holy Karbalaa.

Technical Competencies and member in the IRAQI Engineers Union.

Email:shark_iraq@yahoo.com

Table $1 \mathrm{SMF}$ and DCF parameters

\begin{tabular}{ll}
\hline Parameter & Value \\
\hline SMF & $0.2 \mathrm{~dB} / \mathrm{km}$ \\
Attenuation $(\alpha)$ & $17 \mathrm{ps} / \mathrm{nm} \cdot \mathrm{km}$ \\
Dispersion parameter (D) & $0.075 \mathrm{ps} / \mathrm{nm}^{2} \cdot \mathrm{km}$ \\
Dispersion slope (S) & $80 \mu \mathrm{m}^{2}$ \\
Effective area & $0.1 \mathrm{ps} / \mathrm{km}$ \\
DGD parameter & \\
DCF & $0.5 \mathrm{~dB} / \mathrm{km}$ \\
Attenuation $(\alpha)$ & $-85 \mathrm{ps} / \mathrm{nm}^{-\mathrm{km}}$ \\
Dispersion parameter (D) & $-0.3 \mathrm{ps} / \mathrm{nm}^{2} \cdot \mathrm{km}$ \\
Dispersion slope $(\mathrm{S})$ & $22 \mu \mathrm{m}$ \\
Effective area & $0.1 \mathrm{ps} / \mathrm{km}$ \\
DGD parameter & \\
\hline
\end{tabular}

Table 2 The improvement of $\log { }_{10}$ BER for the channel spacing.

\begin{tabular}{ll}
\hline Channel Spacing (Ghz) & Improvement In Log ${ }_{10}$ Ber At Snr 10 Db \\
\hline $200-100$ & 2.095 \\
$100-50$ & 1.905 \\
$50-25$ & 1.031 \\
\hline
\end{tabular}

Table 3 The improvement of Q-factor for the channel spacing.

\begin{tabular}{ll}
\hline Chanel Spacing (Ghz) & Improvement In Q-Factor At Input Power (P) 5 Dbm \\
\hline $200-100$ & 19.502 \\
$100-50$ & 18.998 \\
$50-25$ & 9.3 \\
\hline
\end{tabular}




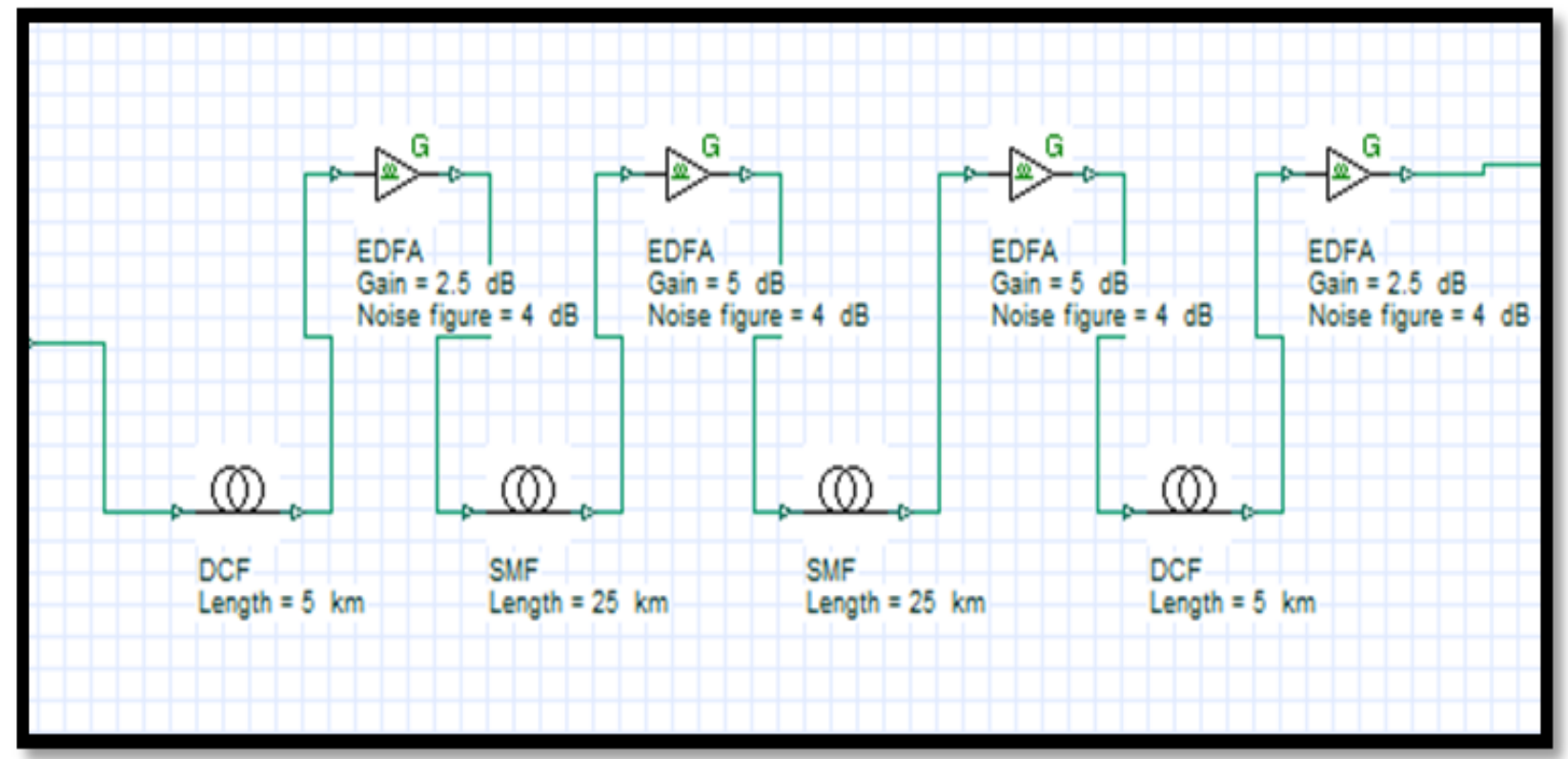

Figure 1 Optical fiber link

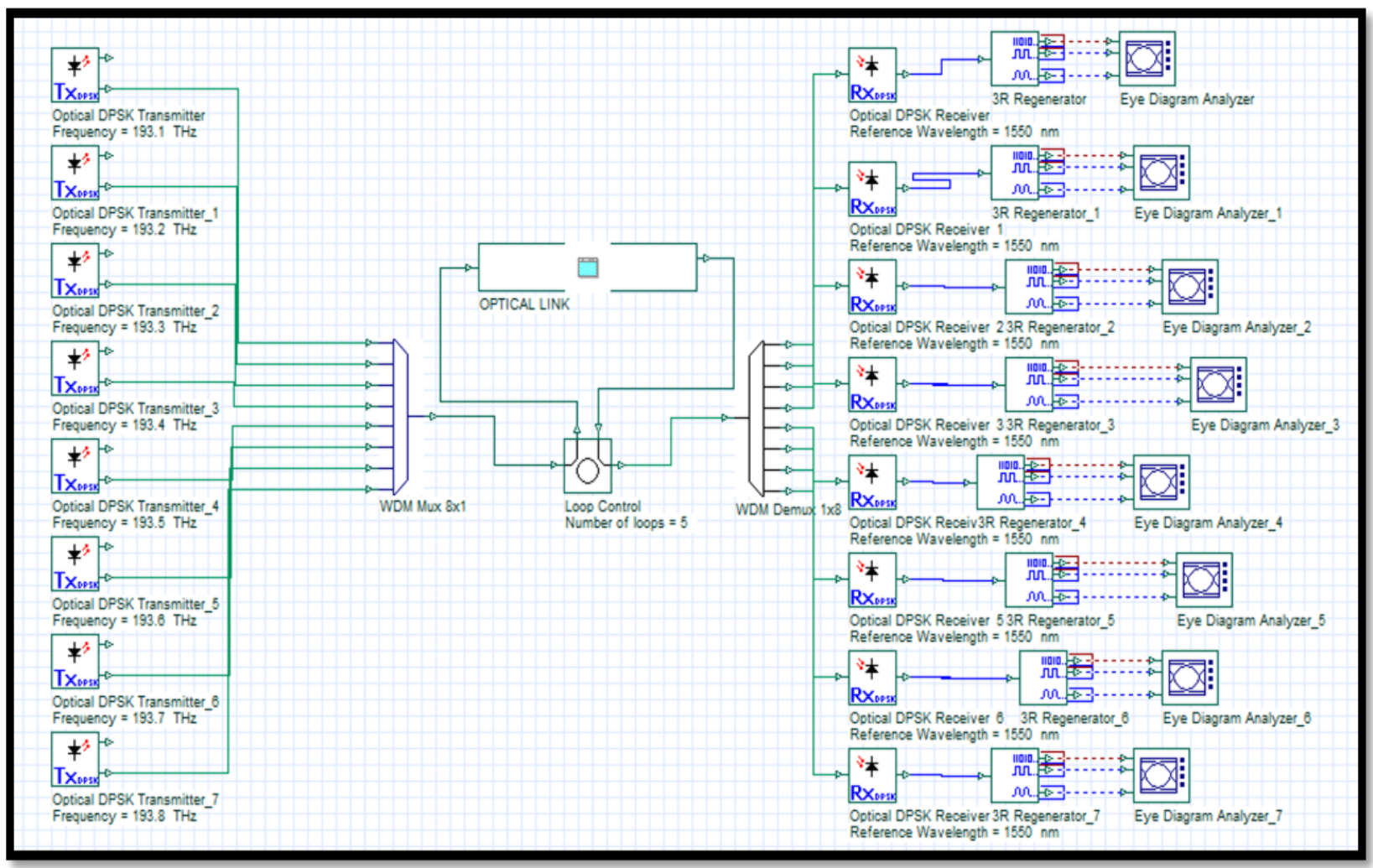

Figure $28 \times 10$ Gbps optical fiber system 


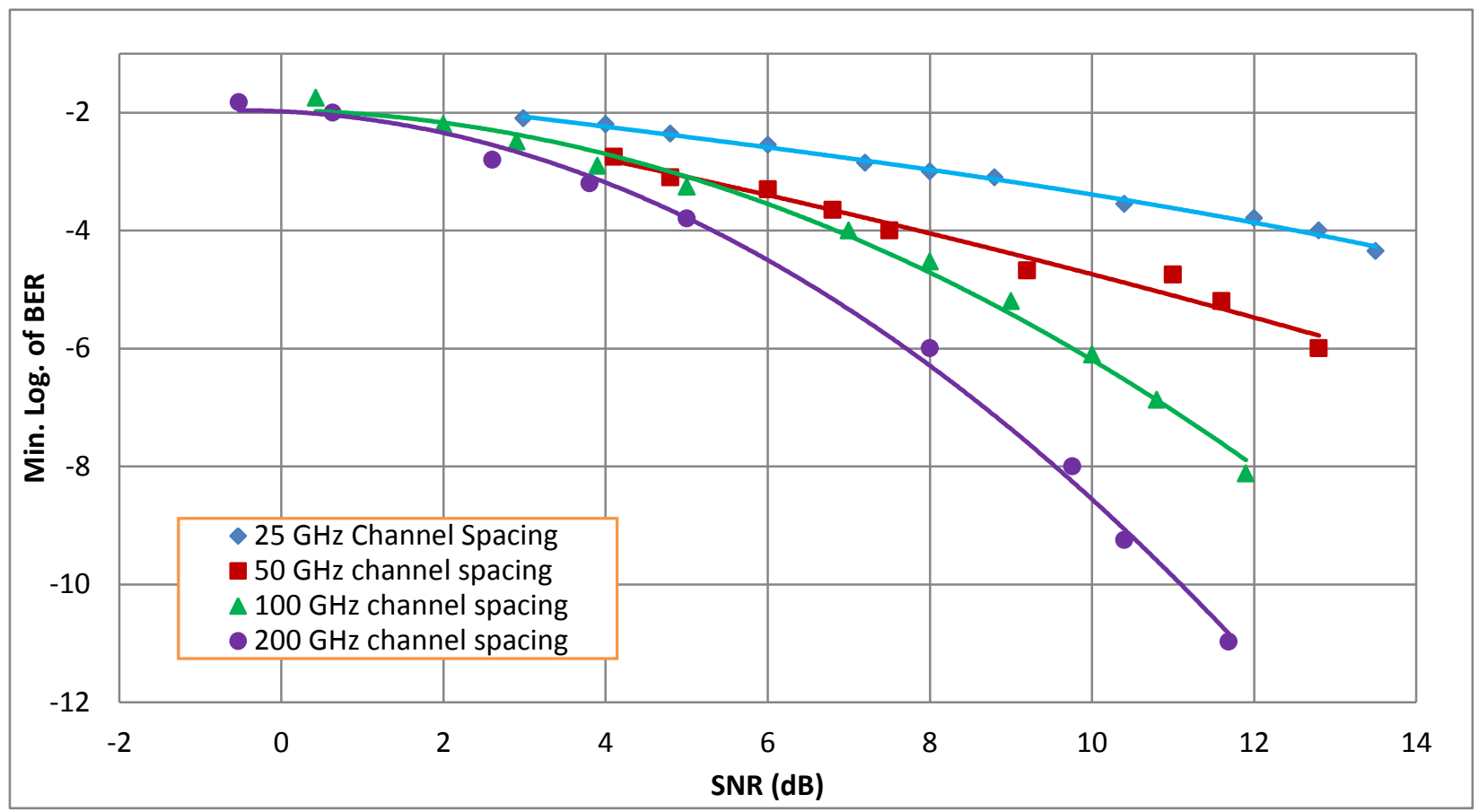

Figure 3 BER versus SNR

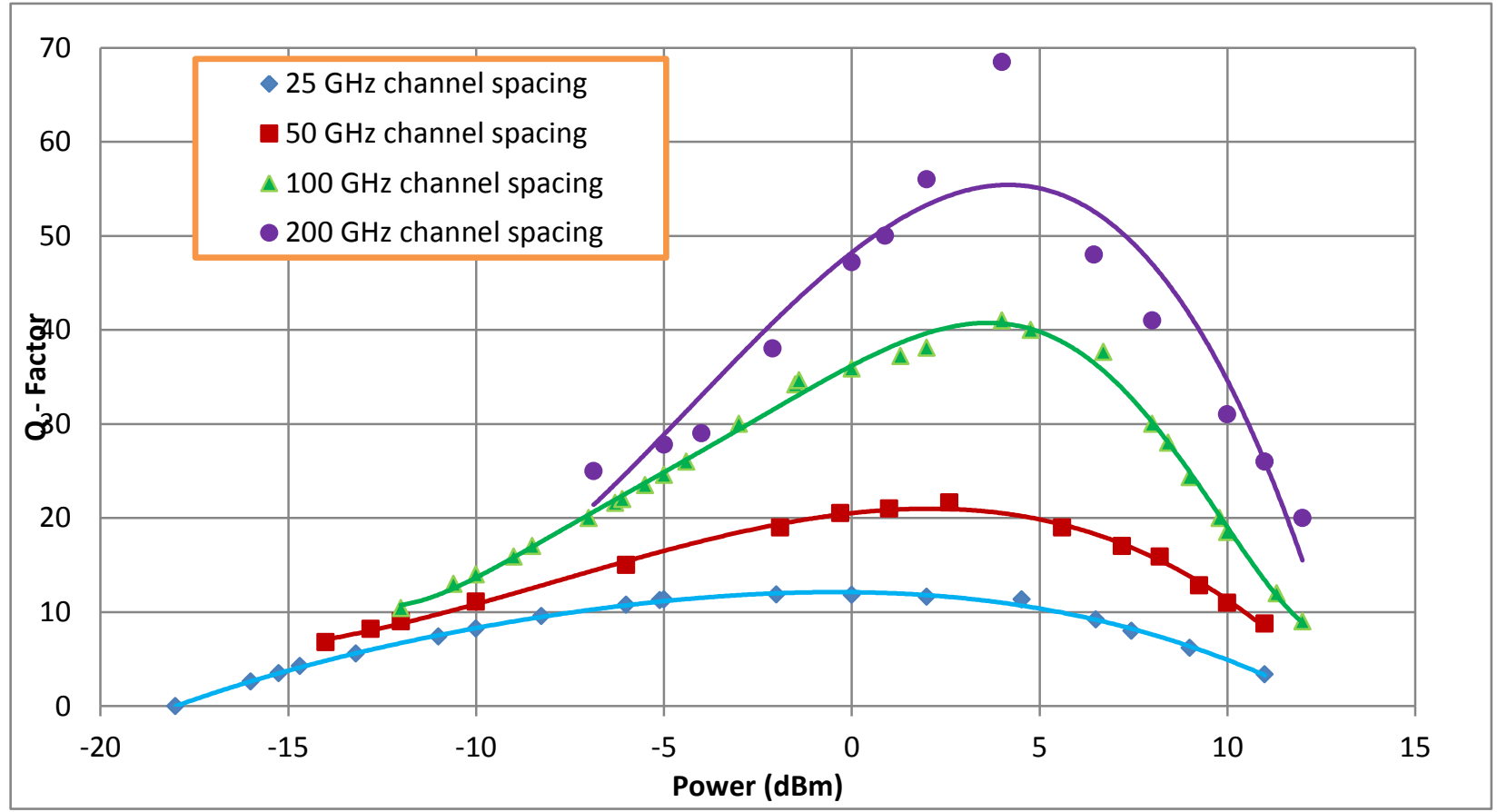

Figure 4 Q - Factor versus input power 
Saif H. Abdulwahed et al.

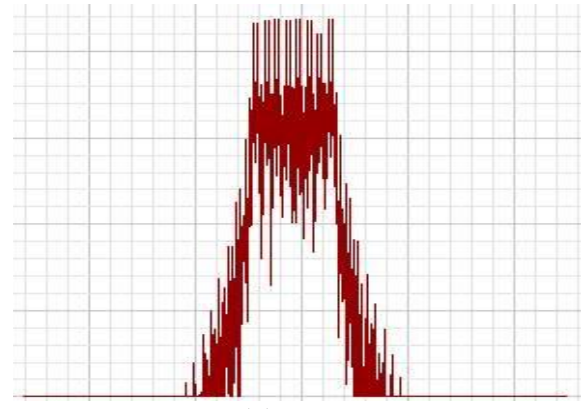

(a)

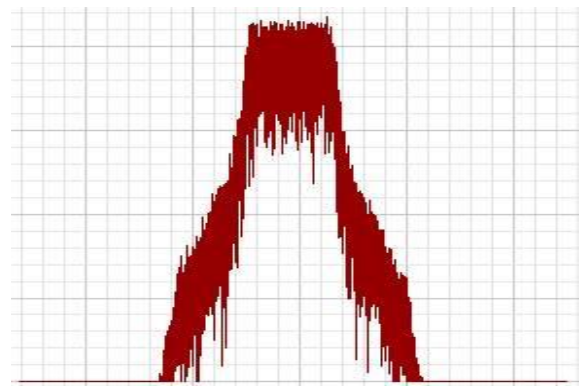

(c)

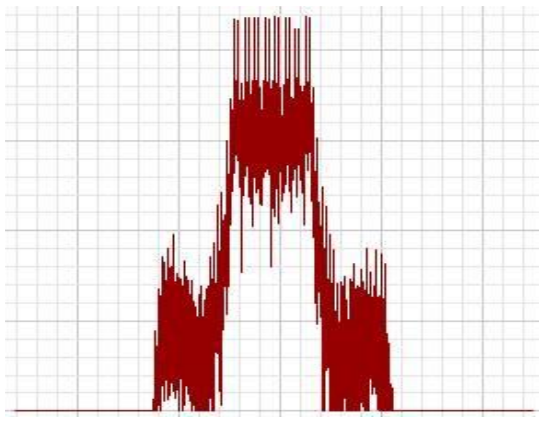

(b)

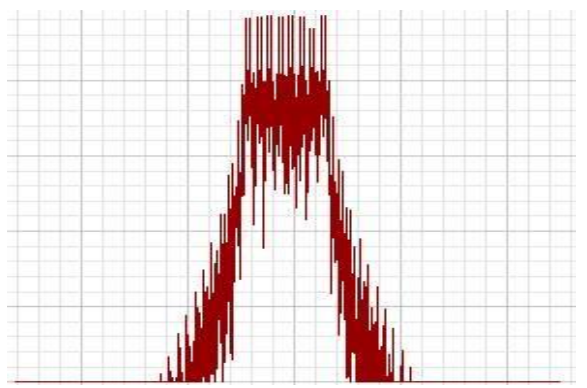

(d)

Figure 5 Spectrum for channel spacing $25 \mathrm{GHz}$ (a) at $0 \mathrm{dBm}$; (b) at $0 \mathrm{dBm}$ after $300 \mathrm{~km}$; (c) at $9 \mathrm{dBm}$; (d) at $9 \mathrm{dBm}$ after $300 \mathrm{~km}$.

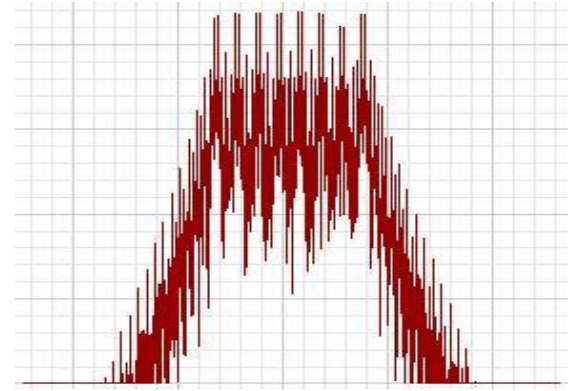

(a)

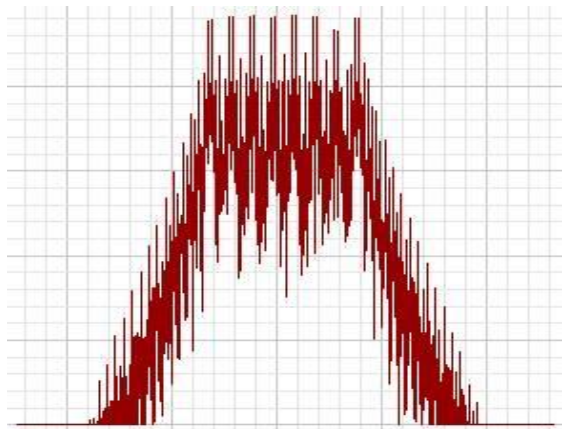

(c)

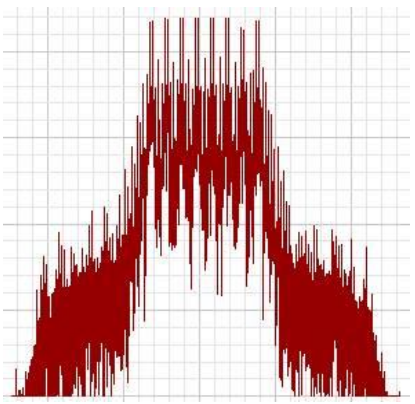

(b)

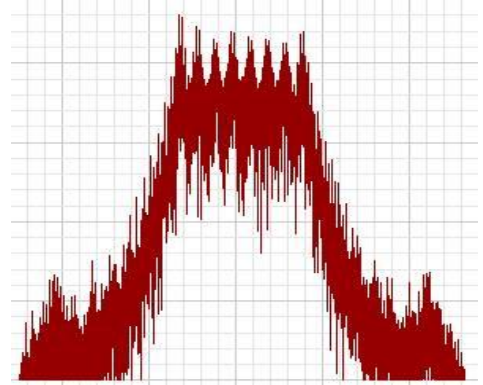

(d)

Figure 6 Spectrums for channel spacing $50 \mathrm{GHz}$

(a) at $0 \mathrm{dBm}$; (b) at $0 \mathrm{dBm}$ after $300 \mathrm{~km}$; (c) at $9 \mathrm{dBm}$; (d) at $9 \mathrm{dBm}$ after $300 \mathrm{~km}$. 